\title{
A q-analogue of Lehmer's congruence
}

by

HaO PAN (Shanghai)

1. Introduction. In 1938, Lehmer [Leh] established an interesting congruence:

$$
\sum_{j=1}^{(p-1) / 2} \frac{1}{j} \equiv-2 \mathrm{Q}_{p}(2)+\mathrm{Q}_{p}(2)^{2} p\left(\bmod p^{2}\right),
$$

where $p \geq 3$ is a prime and $\mathrm{Q}_{p}(2)=\left(2^{p-1}-1\right) / p$. Lehmer's congruence can be considered as an extension of Wolstenholme's [W] harmonic series congruence

$$
\sum_{j=1}^{p-1} \frac{1}{j} \equiv 0\left(\bmod p^{2}\right)
$$

for any prime $p>3$. On the other hand, the $q$-analogues of some arithmetic congruences have been investigated by several authors (e.g., see $[\mathrm{A}],[\mathrm{F}],[\mathrm{C}]$, $[\mathrm{GZ}]$ and $[\mathrm{PS}])$. Recently, Shi and Pan [SP] proved the following $q$-analogue of (1.2):

$$
\sum_{j=1}^{p-1} \frac{1}{[j]_{q}} \equiv \frac{p-1}{2}(1-q)+\frac{p^{2}-1}{24}(1-q)^{2}[p]_{q}\left(\bmod [p]_{q}^{2}\right),
$$

where $[n]_{q}=\left(1-q^{n}\right) /(1-q)=1+q+\cdots+q^{n-1}$. Here congruence (1.3) is considered over the ring of the polynomials in $q$ with integral coefficients. Obviously (1.2) is deduced from (1.3) when $q \rightarrow 1$.

The main purpose of the present paper is to establish a $q$-analogue of Lehmer's congruence. Set

$$
(a ; q)_{n}= \begin{cases}(1-a)(1-a q) \cdots\left(1-a q^{n-1}\right) & \text { if } n \geq 1, \\ 1 & \text { if } n=0 .\end{cases}
$$

2000 Mathematics Subject Classification: Primary 11B65; Secondary 05A10, 05A30, $11 \mathrm{~A} 07$. 
It is easy to see that for any $m \geq 0$ with $p \nmid m$ we have a $q$-analogue of Fermat's little theorem:

$$
\frac{\left(q^{m} ; q^{m}\right)_{p-1}}{(q ; q)_{p-1}} \equiv 1\left(\bmod [p]_{q}\right)
$$

Indeed, since

$$
[m]_{q}=\frac{1-q^{m}}{1-q} \equiv \frac{1-q^{n}}{1-q}=[n]_{q}\left(\bmod [p]_{q}\right)
$$

whenever $m \equiv n(\bmod p)$,

$$
\frac{\left(q^{m} ; q^{m}\right)_{p-1}}{(q ; q)_{p-1}}=\prod_{j=1}^{p-1} \frac{1-q^{j m}}{1-q^{j}}=\prod_{j=1}^{p-1} \frac{[j m]_{q}}{[j]_{q}} \equiv 1\left(\bmod [p]_{q}\right) .
$$

So we can define the $q$-Fermat quotient by

$$
\mathrm{Q}_{p}(m, q)=\frac{\left(q^{m} ; q^{m}\right)_{p-1} /(q ; q)_{p-1}-1}{[p]_{q}} .
$$

TheOrem 1.1. Let $p$ be an odd prime. We have

$$
\begin{aligned}
2 \sum_{j=1}^{(p-1) / 2} \frac{1}{[2 j]_{q}} & +2 \mathrm{Q}_{p}(2, q)-\mathrm{Q}_{p}(2, q)^{2}[p]_{q} \\
& \equiv\left(\mathrm{Q}_{p}(2, q)(1-q)+\frac{p^{2}-1}{8}(1-q)^{2}\right)[p]_{q}\left(\bmod [p]_{q}^{2}\right) .
\end{aligned}
$$

In 1895, with the help of De Moivre's theorem, Morley [M] proved that

$$
(-1)^{(p-1) / 2}\left(\begin{array}{c}
p-1 \\
(p-1) / 2
\end{array}\right) \equiv 4^{p-1}\left(\bmod p^{3}\right)
$$

for any prime $p \geq 5$. In [G1], Granville generalized the congruence of Morley and showed that

$$
(-1)^{(p-1)(m-1) / 2} \prod_{k=1}^{m-1}\left(\begin{array}{c}
p-1 \\
\lfloor k p / m\rfloor
\end{array}\right) \equiv m^{p}-m+1\left(\bmod p^{2}\right)
$$

for any $m \geq 2$ and prime $p \geq 3$, where $\lfloor x\rfloor$ denotes the greatest integer not exceeding $x$. Now we can give the $q$-analogues of (1.6) and (1.7). For any $m, n \in \mathbb{N}$, define the $q$-binomial coefficients by

$$
\left[\begin{array}{l}
n \\
m
\end{array}\right]_{q}=\frac{(q ; q)_{n}}{(q ; q)_{m}(q ; q)_{n-m}}
$$

if $n \geq m$, and if $n<m$, then let $\left[\begin{array}{c}n \\ m\end{array}\right]_{q}=0$. It is easy to see that $\left[\begin{array}{c}n \\ m\end{array}\right]_{q}$ is a polynomial in $q$ with integral coefficients, since the $q$-binomial coefficients 
satisfy the recurrence relation

$$
\left[\begin{array}{c}
n+1 \\
m
\end{array}\right]_{q}=q^{m}\left[\begin{array}{l}
n \\
m
\end{array}\right]_{q}+\left[\begin{array}{c}
n \\
m-1
\end{array}\right]_{q}
$$

THEOREM 1.2 .

$$
\begin{aligned}
(-1)^{(p-1) / 2} q^{\left(p^{2}-1\right) / 4} & {\left[\begin{array}{c}
p-1 \\
(p-1) / 2
\end{array}\right]_{q^{2}} } \\
& \equiv(-q ; q)_{p-1}^{2}-\frac{p^{2}-1}{24}(1-q)^{2}[p]_{q}^{2}\left(\bmod [p]_{q}^{3}\right)
\end{aligned}
$$

for any prime $p \geq 5$.

THEOREM 1.3. Let $p \geq 3$ be a prime and $m \geq 2$ be an integer with $p \nmid m$. Then

$$
\begin{aligned}
&(-1)^{(p-1)(m-1) / 2} q^{M} \prod_{k=1}^{m-1}\left[\begin{array}{c}
p-1 \\
k p / m\rfloor
\end{array}\right]_{q^{m}} \\
& \equiv \frac{m\left(q^{m} ; q^{m}\right)_{p-1}}{(q ; q)_{p-1}}-m+1\left(\bmod [p]_{q}^{2}\right)
\end{aligned}
$$

where

$$
M=m \sum_{k=1}^{m-1}\left(\begin{array}{c}
\lfloor k p / m\rfloor+1 \\
2
\end{array}\right) .
$$

The proofs of Theorems 1.1-1.3 will be given in the next sections.

REMARK. Motivated by the brilliant discovery of Agrawal, Kayal and Saxena $[\mathrm{AKS}]$ on primality testing, the referee posed the interesting problem whether any of these $q$-congruences could identify primes. Recently the author and Chapman established some $q$-analogues of Wilson's theorem [CP]. Hence at least for $p \equiv 3(\bmod 4)$, we have $\prod_{j=1}^{p-1}[j]_{q^{j}} \equiv-1\left(\bmod [p]_{q}\right)$ if and only if $p$ is a prime.

2. Some lemmas. In this section we assume that $p$ is a prime greater than 3. The following lemmas will be used in the proofs of Theorems 1.1 and 1.2 .

LEMMA 2.1.

$$
\begin{aligned}
& \sum_{j=1}^{p-1} \frac{1}{[j]_{q}} \equiv \frac{p-1}{2}(1-q)\left(\bmod [p]_{q}\right), \\
& \sum_{j=1}^{p-1} \frac{q^{j}}{[j]_{q}^{2}} \equiv-\frac{p^{2}-1}{12}(1-q)^{2}\left(\bmod [p]_{q}\right),
\end{aligned}
$$




$$
\sum_{j=1}^{p-1} \frac{1}{[j]_{q}^{2}} \equiv-\frac{(p-1)(p-5)}{12}(1-q)^{2}\left(\bmod [p]_{q}\right)
$$

Proof. See Theorem 4 in [A] and Lemma 2 in [SP].

LEMMA 2.2.

$$
q^{k p}=\sum_{j=0}^{k}(-1)^{j}\left(\begin{array}{l}
k \\
j
\end{array}\right)(1-q)^{j}[p]_{q}^{j}
$$

Proof.

$$
\sum_{j=0}^{k}(-1)^{j}\left(\begin{array}{l}
k \\
j
\end{array}\right)(1-q)^{j}[p]_{q}^{j}=\left(1-(1-q)[p]_{q}\right)^{k}=\left(1-\left(1-q^{p}\right)\right)^{k}=q^{k p}
$$

From Lemma 2.2, we deduce that

$$
q^{k p} \equiv 1-k(1-q)[p]_{q}+\frac{k(k-1)}{2}(1-q)^{2}[p]_{q}^{2}\left(\bmod [p]_{q}^{3}\right)
$$

LEMMA 2.3.

$$
\begin{aligned}
4 \sum_{1 \leq j<k \leq p-1} \frac{(-1)^{k}}{[j]_{q}[k]_{q}} \equiv & \left(\sum_{j=1}^{p-1} \frac{(-1)^{j}}{[j]_{q}}\right)^{2}+(p-3)(1-q) \sum_{k=1}^{p-1} \frac{(-1)^{k}}{[k]_{q}} \\
& +\frac{(p-1)(p+7)}{12}(1-q)^{2}\left(\bmod [p]_{q}\right) .
\end{aligned}
$$

Proof. Since $p$ is odd,

$$
\begin{aligned}
\left(\sum_{j=1}^{p-1} \frac{(-1)^{j}}{[j]_{q}}\right)^{2} & =\left(\sum_{j=1}^{p-1} \frac{(-1)^{j}}{[j]_{q}}\right)\left(\sum_{j=1}^{p-1} \frac{(-1)^{j}}{[j]_{q}}-(1-q) \sum_{j=1}^{p-1}(-1)^{j}\right) \\
& =\left(\sum_{j=1}^{p-1} \frac{(-1)^{j}}{[j]_{q}}\right)\left(\sum_{j=1}^{p-1} \frac{(-q)^{j}}{[j]_{q}}\right) \\
& =\sum_{k=2}^{2 p-2}(-1)^{k} \sum_{j=\max \{1, k-p+1\}}^{\min \{k-1, p-1\}} \frac{q^{j}}{[j]_{q}[k-j]_{q}} .
\end{aligned}
$$

Then we have

$$
\begin{gathered}
\left(\sum_{j=1}^{p-1} \frac{(-1)^{j}}{[j]_{q}}\right)\left(\sum_{j=1}^{p-1} \frac{(-q)^{j}}{[j]_{q}}\right)-(-1)^{p} \sum_{j=1}^{p-1} \frac{q^{j}}{[j]_{q}[p-j]_{q}} \\
=\sum_{k=2}^{p-1}(-1)^{k} \sum_{j=1}^{k-1} \frac{q^{j}}{[j]_{q}[k-j]_{q}}+\sum_{k=p+1}^{2 p-2}(-1)^{k} \sum_{j=k-p+1}^{p-1} \frac{q^{j}}{[j]_{q}[k-j]_{q}}
\end{gathered}
$$




$$
\begin{aligned}
& =\sum_{k=2}^{p-1}(-1)^{k} \sum_{j=1}^{k-1} \frac{q^{j}}{[j]_{q}[k-j]_{q}}+\sum_{l=2}^{p-1}(-1)^{2 p-l} \sum_{j=p-l+1}^{p-1} \frac{q^{j}}{[j]_{q}[2 p-l-j]_{q}} \\
& =\sum_{k=2}^{p-1}(-1)^{k} \sum_{j=1}^{k-1} \frac{q^{j}}{[j]_{q}[k-j]_{q}}+\sum_{l=2}^{p-1}(-1)^{l} \sum_{i=1}^{l-1} \frac{q^{p+i-l}}{[p+i-l]_{q}[p-i]_{q}}
\end{aligned}
$$$$
\text { (here } i=l+j-p \text { ). }
$$

Note that

$$
\begin{aligned}
\frac{q^{p+i-l}}{[p+i-l]_{q}[p-i]_{q}} & =\frac{q^{p+i-l}(1-q)^{2}}{\left(1-q^{p+i-l}\right)\left(1-q^{p-i}\right)} \\
& \equiv \frac{q^{i}(1-q)^{2}}{\left(1-q^{l-i}\right)\left(1-q^{i}\right)}\left(\bmod [p]_{q}\right)
\end{aligned}
$$

It follows that

$$
\begin{aligned}
\left(\sum_{j=1}^{p-1} \frac{(-1)^{j}}{[j]_{q}}\right) & \left(\sum_{j=1}^{p-1} \frac{(-q)^{j}}{[j]_{q}}\right)+\sum_{j=1}^{p-1} \frac{q^{j}}{[j]_{q}[p-j]_{q}} \\
& \equiv \sum_{k=2}^{p-1}(-1)^{k} \sum_{j=1}^{k-1} \frac{q^{j}}{[j]_{q}[k-j]_{q}}+\sum_{l=2}^{p-1}(-1)^{l} \sum_{i=1}^{l-1} \frac{q^{i}}{[i]_{q}[l-i]_{q}} \\
& =2 \sum_{k=2}^{p-1}(-1)^{k} \sum_{j=1}^{k-1} \frac{q^{j}(1-q)^{2}}{\left(1-q^{j}\right)\left(1-q^{k-j}\right)} \\
& =2 \sum_{k=2}^{p-1} \frac{(-1)^{k} q^{k}(1-q)^{2}}{1-q^{k}} \sum_{j=1}^{k-1}\left(\frac{1}{q^{k-j}-q^{k}}+\frac{1}{1-q^{k-j}}\right) \\
& =2 \sum_{1 \leq j<k \leq p-1} \frac{(-1)^{k}\left(q^{k}+q^{j}\right)}{[j]_{q}[k]_{q}}\left(\bmod [p]_{q}\right) .
\end{aligned}
$$

We can write

$$
\begin{aligned}
& \sum_{1 \leq j<k \leq p-1} \frac{(-1)^{k}\left(q^{k}+q^{j}\right)}{[j]_{q}[k]_{q}} \\
= & \sum_{1 \leq j<k \leq p-1} \frac{(-1)^{k}\left(2-\left(1-q^{k}\right)-\left(1-q^{j}\right)\right)}{[j]_{q}[k]_{q}} \\
= & 2 \sum_{1 \leq j<k \leq p-1} \frac{(-1)^{k}}{[j]_{q}[k]_{q}}-(1-q)\left(\sum_{k=2}^{p-1} \frac{(-1)^{k}(k-1)}{[k]_{q}}+\sum_{j=1}^{p-2} \frac{1}{[j]_{q}} \sum_{k=j+1}^{p-1}(-1)^{k}\right) .
\end{aligned}
$$


Now

$$
\begin{aligned}
\sum_{k=2}^{p-1} \frac{(-1)^{k}(k-1)}{[k]_{q}}=\frac{1}{2} \sum_{k=1}^{p-1}\left(\frac{(-1)^{k}(k-1)}{[k]_{q}}+\frac{(-1)^{p-k}(p-k-1)}{[p-k]_{q}}\right) \\
=\frac{1}{2} \sum_{k=1}^{p-1}(-1)^{k}(k-1)\left(\frac{1}{[k]_{q}}+\frac{1}{[p-k]_{q}}\right)+\frac{p-2}{2} \sum_{k=1}^{p-1} \frac{(-1)^{k}}{[k]_{q}} \\
=\frac{1}{2} \sum_{k=1}^{p-1}(-1)^{k}(k-1)\left(\frac{[p]_{q}}{[k][p-k]_{q}}+(1-q)\right)+\frac{p-2}{2} \sum_{k=1}^{p-1} \frac{(-1)^{k}}{[k]_{q}} \\
\equiv \frac{p-1}{4}(1-q)+\frac{p-2}{2} \sum_{k=1}^{p-1} \frac{(-1)^{k}}{[k]_{q}}\left(\bmod [p]_{q}\right) .
\end{aligned}
$$

And from (2.1) we have

$$
\begin{aligned}
\sum_{j=1}^{p-2} \frac{1}{[j]_{q}} \sum_{k=j+1}^{p-1}(-1)^{k} & =\frac{1}{2} \sum_{j=1}^{p-1} \frac{1-(-1)^{j}}{[j]_{q}} \\
& \equiv \frac{p-1}{4}(1-q)-\frac{1}{2} \sum_{j=1}^{p-1} \frac{(-1)^{j}}{[j]_{q}}\left(\bmod [p]_{q}\right) .
\end{aligned}
$$

Finally, by (2.3),

$$
\begin{aligned}
\sum_{j=1}^{p-1} \frac{q^{j}}{[j]_{q}[p-j]_{q}} & =\sum_{j=1}^{p-1} \frac{q^{p-j}}{[j]_{q}[p-j]_{q}}=\sum_{j=1}^{p-1} \frac{q^{p}}{[j]_{q}\left([p]_{q}-[j]_{q}\right)} \\
& \equiv \frac{(p-1)(p-5)}{12}(1-q)^{2}\left(\bmod [p]_{q}\right) .
\end{aligned}
$$

Thus combining the equations and congruences above, we obtain

$$
\begin{aligned}
& 4 \sum_{1 \leq j<k \leq p-1}(-1)^{k} \frac{1}{[j]_{q}[k]_{q}}-\left(\sum_{j=1}^{p-1} \frac{(-1)^{j}}{[j]_{q}}\right)\left(\sum_{j=1}^{p-1} \frac{(-q)^{j}}{[j]_{q}}\right) \\
& \equiv(p-3)(1-q) \sum_{k=1}^{p-1} \frac{(-1)^{k}}{[k]_{q}}+\frac{(p-1)(p+7)}{12}(1-q)^{2}\left(\bmod [p]_{q}\right) .
\end{aligned}
$$

Lemma 2.4 .

$$
\begin{aligned}
\sum_{j=1}^{p-1} \frac{(-1)^{j}}{[j]_{q}} \equiv & 2 \sum_{j=1}^{(p-1) / 2} \frac{1}{[2 j]_{q}}-\frac{p-1}{2}(1-q) \\
& -\frac{p^{2}-1}{24}(1-q)^{2}[p]_{q}\left(\bmod [p]_{q}^{2}\right) .
\end{aligned}
$$


Proof. Clearly

$$
\begin{aligned}
\sum_{j=1}^{p-1} \frac{(-1)^{j}}{[j]_{q}} & =\sum_{j=1}^{(p-1) / 2} \frac{1}{[2 j]_{q}}-\sum_{j=1}^{(p-1) / 2} \frac{1}{[2 j-1]_{q}} \\
& =\sum_{j=1}^{(p-1) / 2} \frac{1}{[2 j]_{q}}-\sum_{j=1}^{(p-1) / 2} \frac{1}{[p-2 j]_{q}} .
\end{aligned}
$$

Observe that

$$
\begin{aligned}
\frac{1}{[p-2 j]_{q}} & =\frac{q^{2 j}}{[p]_{q}-[2 j]_{q}}=\frac{q^{2 j}\left([p]_{q}+[2 j]_{q}\right)}{[p]_{q}^{2}-[2 j]_{q}^{2}} \\
& \equiv-\frac{q^{2 j}\left([p]_{q}+[2 j]_{q}\right)}{[2 j]_{q}^{2}}\left(\bmod [p]_{q}^{2}\right)
\end{aligned}
$$

By (2.2), we have

$$
\begin{aligned}
-\frac{p^{2}-1}{12}(1-q)^{2} & \equiv \sum_{j=1}^{p-1} \frac{q^{j}}{[j]_{q}^{2}}=\sum_{j=1}^{(p-1) / 2} \frac{q^{2 j}}{[2 j]_{q}^{2}}+\sum_{j=1}^{(p-1) / 2} \frac{q^{p-2 j}}{[p-2 j]_{q}^{2}} \\
& =\sum_{j=1}^{(p-1) / 2} \frac{q^{2 j}}{[2 j]_{q}^{2}}+\sum_{j=1}^{(p-1) / 2} \frac{q^{p+2 j}}{\left([p]_{q}-[2 j]_{q}\right)^{2}} \\
& \equiv 2 \sum_{j=1}^{(p-1) / 2} \frac{q^{2 j}}{[2 j]_{q}^{2}}\left(\bmod [p]_{q}\right) .
\end{aligned}
$$

Hence

$$
\begin{aligned}
\sum_{j=1}^{p-1} \frac{(-1)^{j}}{[j]_{q}} & \equiv \sum_{j=1}^{(p-1) / 2} \frac{1}{[2 j]_{q}}+\sum_{j=1}^{(p-1) / 2} \frac{q^{2 j}\left([p]_{q}+[2 j]_{q}\right)}{[2 j]_{q}^{2}} \\
& \equiv \sum_{j=1}^{(p-1) / 2} \frac{1+q^{2 j}}{[2 j]_{q}}-\frac{p^{2}-1}{24}(1-q)^{2}[p]_{q} \\
& =2 \sum_{j=1}^{(p-1) / 2} \frac{1}{[2 j]_{q}}-\frac{p-1}{2}(1-q)-\frac{p^{2}-1}{24}(1-q)^{2}[p]_{q}\left(\bmod [p]_{q}^{2}\right)
\end{aligned}
$$

\section{Proofs of Theorems 1.1 and $\mathbf{1 . 2}$}

Proof of Theorem 1.1. One can directly verify (1.5) when $p=3$. So below we assume that $p \geq 5$. It is well-known (cf. Corollary 10.2.2 of [AAR]) that

$$
(x ; q)_{n}=\sum_{j=0}^{n}\left[\begin{array}{l}
n \\
j
\end{array}\right]_{q} q^{\left(\begin{array}{c}
j \\
2
\end{array}\right)}(-x)^{j} .
$$


Then we have

$$
\begin{aligned}
& (3.1) \quad \frac{(-1 ; q)_{p}-q^{\left(\begin{array}{c}
p \\
2
\end{array}\right)}-1}{[p]_{q}} \\
& =\frac{1}{[p]_{q}} \sum_{k=1}^{p-1}\left[\begin{array}{l}
p \\
k
\end{array}\right]_{q} q^{\left(\begin{array}{l}
k \\
2
\end{array}\right)}=\sum_{k=1}^{p-1} \frac{1}{[k]_{q}} \prod_{j=1}^{k-1} \frac{q^{j}\left(1-q^{p-j}\right)}{1-q^{j}}
\end{aligned}
$$$$
=\sum_{k=1}^{p-1} \frac{1}{[k]_{q}} \prod_{j=1}^{k-1}\left(\frac{[p]_{q}}{[j]_{q}}-1\right) \equiv[p]_{q} \sum_{1 \leq j<k \leq p-1} \frac{(-1)^{k}}{[j]_{q}[k]_{q}}-\sum_{k=1}^{p-1} \frac{(-1)^{k}}{[k]_{q}}\left(\bmod [p]_{q}^{2}\right) .
$$

Consequently,

$$
\begin{aligned}
\sum_{k=1}^{p-1} \frac{(-1)^{k}}{[k]_{q}} & \equiv-\frac{(-1 ; q)_{p}-q^{\left(\begin{array}{c}
p \\
2
\end{array}\right)}-1}{[p]_{q}} \\
& \equiv-\frac{2(-q ; q)_{p-1}-2}{[p]_{q}}-\frac{p-1}{2}(1-q)\left(\bmod [p]_{q}\right) .
\end{aligned}
$$

Thus, applying Lemma 2.3, we have

$$
\begin{aligned}
& \sum_{1 \leq j<k \leq p-1} \frac{(-1)^{k}}{[j]_{q}[k]_{q}}-\frac{(p-1)(p+7)}{48}(1-q)^{2} \\
\equiv & \frac{1}{4}\left(\sum_{k=1}^{p-1} \frac{(-1)^{k}}{[k]_{q}}\right)\left(\sum_{k=1}^{p-1} \frac{(-1)^{k}}{[k]_{q}}+(p-3)(1-q)\right) \\
\equiv & \frac{1}{4}\left(-2 \mathrm{Q}_{p}(2, q)-\frac{p-1}{2}(1-q)\right)\left(-2 \mathrm{Q}_{p}(2, q)+\frac{p-5}{2}(1-q)\right) \\
= & \mathrm{Q}_{p}(2, q)^{2}+\mathrm{Q}_{p}(2, q)(1-q)-\frac{(p-1)(p-5)}{16}(1-q)^{2}\left(\bmod [p]_{q}\right) .
\end{aligned}
$$

On the other hand, it follows from (2.4) that

$$
\begin{aligned}
& \frac{(-1 ; q)_{p}-q^{\left(\begin{array}{c}
p \\
2
\end{array}\right)}-1}{[p]_{q}} \\
& \equiv \frac{2(-q ; q)_{p-1}-2}{[p]_{q}}+\frac{p-1}{2}(1-q)-\frac{(p-1)(p-3)}{8}(1-q)^{2}[p]_{q}\left(\bmod [p]_{q}^{2}\right) .
\end{aligned}
$$

Then by Lemma 2.4,

$$
\begin{aligned}
& \sum_{k=1}^{p-1} \frac{(-1)^{k}}{[k]_{q}}+\frac{(-1 ; q)_{p}-q^{\left(\begin{array}{c}
p \\
2
\end{array}\right)}-1}{[p]_{q}} \\
\equiv & 2 \sum_{j=1}^{(p-1) / 2} \frac{1}{[2 j]_{q}}+2 \mathrm{Q}_{p}(2, q)-\frac{(p-1)(p-2)}{6}(1-q)^{2}[p]_{q}\left(\bmod [p]_{q}^{2}\right) .
\end{aligned}
$$

Combining (3.1), (3.2) and (3.3), the desired (1.5) is obtained. 
Proof of Theorem 1.2. Since

$$
\left[\begin{array}{c}
p-1 \\
(p-1) / 2
\end{array}\right]_{q^{2}}=\prod_{j=1}^{(p-1) / 2} \frac{[p-j]_{q^{2}}}{[j]_{q^{2}}}=\prod_{j=1}^{(p-1) / 2} \frac{[p]_{q^{2}}-[j]_{q^{2}}}{q^{2 j}[j]_{q^{2}}}
$$

we have

$$
\begin{aligned}
& (-1)^{(p-1) / 2} q^{\left(p^{2}-1\right) / 4}\left[\begin{array}{c}
p-1 \\
(p-1) / 2
\end{array}\right]_{q^{2}}=\prod_{j=1}^{(p-1) / 2}\left(1-\frac{[p]_{q^{2}}}{[j]_{q^{2}}}\right) \\
& \equiv 1-\frac{1+q^{p}}{1+q} \sum_{j=1}^{(p-1) / 2} \frac{[p]_{q}}{[j]_{q^{2}}}+\frac{\left(1+q^{p}\right)^{2}}{(1+q)^{2}} \sum_{1 \leq j<k \leq(p-1) / 2} \frac{[p]_{q}^{2}}{[j]_{q^{2}}[k]_{q^{2}}}\left(\bmod [p]_{q^{2}}^{3}\right) .
\end{aligned}
$$

From Theorem 1.1, we deduce that

$$
\begin{aligned}
\frac{1+q^{p}}{1+q} & \sum_{j=1}^{(p-1) / 2} \frac{1}{[j]_{q^{2}}}=\left(1+q^{p}\right) \sum_{j=1}^{(p-1) / 2} \frac{1}{[2 j]_{q}} \\
\equiv & -\left(1+q^{p}\right) \mathrm{Q}_{p}(2, q) \\
& +\frac{1+q^{p}}{2}\left(\mathrm{Q}_{p}(2, q)^{2}+\mathrm{Q}_{p}(2, q)(1-q)+\frac{p^{2}-1}{8}(1-q)^{2}\right)[p]_{q} \\
\equiv & -\left(1+q^{p}\right) \mathrm{Q}_{p}(2, q)+\mathrm{Q}_{p}(2, q)^{2}[p]_{q}+\mathrm{Q}_{p}(2, q)(1-q)[p]_{q} \\
& +\frac{p^{2}-1}{8}(1-q)^{2}[p]_{q}\left(\bmod [p]_{q}^{2}\right) .
\end{aligned}
$$

Notice that

$$
\begin{aligned}
\sum_{1 \leq j<k \leq(p-1) / 2} \frac{1}{[j]_{q^{2}}[k]_{q^{2}}} & =\frac{1}{2}\left(\left(\sum_{j=1}^{(p-1) / 2} \frac{1}{[j]_{q^{2}}}\right)^{2}-\sum_{j=1}^{(p-1) / 2} \frac{1}{[j]_{q^{2}}^{2}}\right) \\
& =\frac{(1+q)^{2}}{2}\left(\left(\sum_{j=1}^{(p-1) / 2} \frac{1}{[2 j]_{q}}\right)^{2}-\sum_{j=1}^{(p-1) / 2} \frac{1}{[2 j]_{q}^{2}}\right) .
\end{aligned}
$$

Theorem 1.1 implies that

$$
\sum_{j=1}^{(p-1) / 2} \frac{1}{[2 j]_{q}} \equiv-\mathrm{Q}_{p}(2, q)\left(\bmod [p]_{q}\right) .
$$

Then using (2.7), we get

$$
\begin{aligned}
\sum_{j=1}^{(p-1) / 2} \frac{1}{[2 j]_{q}^{2}} & =\sum_{j=1}^{(p-1) / 2} \frac{q^{2 j}}{[2 j]_{q}^{2}}+(1-q) \sum_{j=1}^{(p-1) / 2} \frac{1}{[2 j]_{q}} \\
& \equiv-\frac{p^{2}-1}{24}(1-q)^{2}-\mathrm{Q}_{p}(2, q)(1-q)\left(\bmod [p]_{q}\right)
\end{aligned}
$$


Consequently,

$$
\begin{aligned}
& \frac{2}{(1+q)^{2}} \sum_{1 \leq j<k \leq(p-1) / 2} \frac{1}{[j]_{q^{2}}[k]_{q^{2}}} \\
& \quad \equiv \mathrm{Q}_{p}(2, q)^{2}+\mathrm{Q}_{p}(2, q)(1-q)+\frac{p^{2}-1}{24}(1-q)^{2}\left(\bmod [p]_{q}\right) .
\end{aligned}
$$

Thus it follows from (3.4), (3.5) and (3.6) that

$$
\begin{aligned}
& (-1)^{(p-1) / 2} q^{\left(p^{2}-1\right) / 4}\left[\begin{array}{c}
p-1 \\
(p-1) / 2
\end{array}\right]_{q^{2}}-1 \\
& \equiv[p]_{q}^{2} \frac{4}{(1+q)^{2}} \sum_{1 \leq j<k \leq(p-1) / 2} \frac{1}{[j]_{q^{2}}[k]_{q^{2}}}-[p]_{q} \frac{1+q^{p}}{1+q} \sum_{j=1}^{(p-1) / 2} \frac{1}{[j]_{q^{2}}} \\
& \equiv \mathrm{Q}_{p}(2, q)^{2}[p]_{q}^{2}+\left(1+q^{p}\right) \mathrm{Q}_{p}(2, q)[p]_{q}+\mathrm{Q}_{p}(2, q)(1-q)[p]_{q}^{2}-\frac{p^{2}-1}{24}(1-q)^{2}[p]_{q}^{2} \\
& =\left((-q ; q)_{p-1}-1\right)\left((-q ; q)_{p-1}+1\right)-\frac{p^{2}-1}{24}(1-q)^{2}[p]_{q}^{2}\left(\bmod [p]_{q}^{3}\right) .
\end{aligned}
$$

\section{Fermat quotient}

Lemma 4.1. Let $p$ be an odd prime. Suppose that $m$ is a positive integer with $(m, p)=1$. Then

$$
\mathrm{Q}_{p}(m, q) \equiv \sum_{j=1}^{p-1} \frac{\lfloor j m / p\rfloor}{[j m]_{q}}-\frac{(p-1)(m-1)}{2}(1-q)\left(\bmod [p]_{q}\right) .
$$

Proof. For each $j \in\{1, \ldots, p-1\}$, let $r_{j}=j m-\lfloor j m / p\rfloor p$. Then

$$
\begin{aligned}
\frac{\left(q^{m} ; q^{m}\right)_{p-1}}{(q ; q)_{p-1}} & =\prod_{j=1}^{p-1} \frac{1-q^{j m}}{1-q^{j}}=\prod_{j=1}^{p-1}\left(\frac{1-q^{r_{j}}}{1-q^{j}}+\frac{q^{r_{j}}\left(1-q^{\lfloor j m / p\rfloor p}\right)}{1-q^{j}}\right) \\
& =\prod_{j=1}^{p-1} \frac{1-q^{r_{j}}}{1-q^{j}}\left(1+\frac{q^{r_{j}}\left(1-q^{\lfloor j m / p\rfloor p}\right)}{1-q^{r_{j}}}\right) .
\end{aligned}
$$

Since $r_{j}$ runs through $1, \ldots, p-1$ together with $j$, we have

$$
\begin{aligned}
& \frac{\left(q^{m} ; q^{m}\right)_{p-1}}{(q ; q)_{p-1}} \\
& \quad=\prod_{j=1}^{p-1}\left(1+\frac{q^{r_{j}}\left(1-q^{\lfloor j m / p\rfloor p}\right)}{1-q^{r_{j}}}\right) \equiv 1+\left(1-q^{p}\right) \sum_{j=1}^{p-1} \frac{q^{r_{j}}}{1-q^{r_{j}}} \cdot \frac{1-q^{\lfloor j m / p\rfloor p}}{1-q^{p}} \\
& \quad \equiv 1+\left(1-q^{p}\right) \sum_{j=1}^{p-1}\left\lfloor\frac{j m}{p}\right\rfloor \frac{q^{r_{j}}}{1-q^{r_{j}}} \equiv 1+[p]_{q} \sum_{j=1}^{p-1}\left\lfloor\frac{j m}{p}\right\rfloor \frac{q^{j m}}{[j m]_{q}}\left(\bmod [p]_{q}^{2}\right) .
\end{aligned}
$$


Finally,

$$
\begin{aligned}
\sum_{j=1}^{p-1}\left\lfloor\frac{j m}{p}\right\rfloor \frac{q^{j m}}{[j m]_{q}} & =\sum_{j=1}^{p-1} \frac{\lfloor j m / p\rfloor}{\lfloor j m]_{q}}-(1-q) \sum_{j=1}^{p-1}\left\lfloor\frac{j m}{p}\right\rfloor \\
& =\sum_{j=1}^{p-1} \frac{\lfloor j m / p\rfloor}{\lfloor j m]_{q}}-\frac{(p-1)(m-1)}{2}(1-q) .
\end{aligned}
$$

REMARK. Letting $q \rightarrow 1$ in (4.1), we obtain

$$
\frac{m^{p}-m}{p} \equiv \sum_{j=1}^{p-1} \frac{\lfloor j m / p\rfloor}{j}(\bmod p)
$$

which was first discovered by Lerch [Ler].

Proof of Theorem 1.3. We write

$$
\left.\left[\begin{array}{c}
p-1 \\
\lfloor k p / m\rfloor
\end{array}\right]_{q^{m}}=\prod_{j=1}^{\lfloor k p / m\rfloor} \frac{[p]_{q^{m}}-[j]_{q^{m}}}{q^{j m}[j]_{q^{m}}}=q^{-m(\lfloor k p / m\rfloor+1}\right) \prod_{j=1}^{\lfloor k p / m\rfloor}\left(\frac{[p]_{q^{m}}}{[j]_{q^{m}}}-1\right) .
$$

As $p \nmid m,[p]_{q}$ divides $[p]_{q^{m}}=\left(1-q^{m p}\right) /\left(1-q^{m}\right)$. Thus

$$
\begin{aligned}
& (-1)^{(p-1)(m-1) / 2} q^{\sum_{k=1}^{m-1} m\left(\begin{array}{c}
\lfloor k p / m\rfloor+1 \\
2
\end{array}\right)} \prod_{k=1}^{m-1}\left[\begin{array}{c}
p-1 \\
\lfloor k p / m\rfloor
\end{array}\right]_{q^{m}} \\
& =\prod_{k=1}^{m-1} \prod_{j=1}^{\lfloor k p / m\rfloor}\left(1-\frac{[p]_{q^{m}}}{[j]_{q^{m}}}\right) \\
& \left.\equiv 1-[p]_{q^{m}} \sum_{k=1}^{m-1} \sum_{1 \leq j<k p / m} \frac{1}{[j]_{q^{m}}} \quad \text { (here } k p / m \notin \mathbb{Z}, \text { so } j \leq\lfloor k p / m\rfloor<k p / m\right) \\
& =1-[p]_{q^{m}} \sum_{j=1}^{p-1} \frac{m-1-\lfloor j m / p\rfloor}{[j]_{q^{m}}}\left(\bmod [p]_{q}^{2}\right) .
\end{aligned}
$$

In view of (2.1) and Lemma 4.1, we have

$$
\begin{aligned}
& {[p]_{q^{m}} \sum_{j=1}^{p-1} \frac{m-1-\lfloor j m / p\rfloor}{[j]_{q^{m}}}} \\
& \quad=(m-1)[m p]_{q} \sum_{j=1}^{p-1} \frac{1}{[j m]_{q}}-[m p]_{q} \sum_{j=1}^{p-1} \frac{\lfloor j m / p\rfloor}{[j m]_{q}} \\
& \quad \equiv(m-1)[m p]_{q} \frac{p-1}{2}(1-q)-[m p]_{q} \mathrm{Q}_{p}(m, q)-\frac{(p-1)(m-1)}{2}(1-q)[m p]_{q} \\
& \quad \equiv-m[p]_{q} \mathrm{Q}_{p}(m, q)\left(\bmod [p]_{q}^{2}\right) .
\end{aligned}
$$


REMARK. For further developments of Granville's congruence (1.7), the reader is referred to $[\mathrm{S}]$.

5. A conjecture of Skula. Recently, with the help of polynomials over finite fields, Granville [G2] confirmed a conjecture of Skula:

$$
\left(\frac{2^{p-1}-1}{p}\right)^{2} \equiv-\sum_{j=1}^{p-1} \frac{2^{j}}{j^{2}}(\bmod p)
$$

for any prime $p \geq 5$. Using our $q$-analogue of Lehmer's congruence, we have the following $q$-analogue of (5.1):

Theorem 5.1. Let $p \geq 5$ be a prime. Then

$$
\begin{aligned}
& \sum_{j=1}^{p-1} \frac{q^{j}(-q ; q)_{j}}{[j]_{q}^{2}}+\mathrm{Q}_{p}(2, q)^{2} \\
& \equiv-(p-1) \mathrm{Q}_{p}(2, q)(1-q)-\frac{(7 p-5)(p-1)}{24}(1-q)^{2}\left(\bmod [p]_{q}\right) .
\end{aligned}
$$

Lemma 5.2.

$$
\sum_{k=0}^{n}(-1)^{k}\left[\begin{array}{l}
n \\
k
\end{array}\right]_{q} q^{\left(\begin{array}{c}
n-k \\
2
\end{array}\right)}(-q ; q)_{k}=(-1)^{n} q^{\left(\begin{array}{c}
n+1 \\
2
\end{array}\right)}
$$

Proof. From the well-known $q$-binomial theorem (cf. Theorem 10.2.1 of $[\mathrm{AAR}])$, we have

$$
\sum_{k=0}^{\infty} \frac{(-1)^{k} q^{\left(\begin{array}{c}
k \\
2
\end{array}\right)}}{(q ; q)_{k}} x^{k}=(x ; q)_{\infty} \quad \text { and } \quad \sum_{k=0}^{\infty} \frac{(-q ; q)_{k}}{(q ; q)_{k}} x^{k}=\frac{(-q x ; q)_{\infty}}{(x ; q)_{\infty}} .
$$

Then by comparing the coefficient of $x^{n}$ on both sides of

$$
(x ; q)_{\infty} \frac{(-q x ; q)_{\infty}}{(x ; q)_{\infty}}=(-q x ; q)_{\infty},
$$

we obtain

$$
\sum_{k=0}^{n} \frac{(-1)^{n-k} q^{\left(\begin{array}{c}
n-k \\
2
\end{array}\right)}(-q ; q)_{k}}{(q ; q)_{n-k}(q ; q)_{k}}=\frac{q^{\left(\begin{array}{c}
n \\
2
\end{array}\right)+n}}{(q ; q)_{n}}
$$

which is an equivalent form of (5.3).

Corollary 5.3. For any odd prime $p$, we have

$$
\sum_{j=1}^{p-1} \frac{q^{j}(-q ; q)_{j}}{[j]_{q}} \equiv-2 \mathrm{Q}_{p}(2, q)-(p-1)(1-q)\left(\bmod [p]_{q}\right) .
$$


Proof. From Lemma 5.2, we deduce that

$$
\begin{aligned}
\sum_{j=1}^{p-1} \frac{q^{j}(-q ; q)_{j}}{[j]_{q}} & \equiv \sum_{j=1}^{p-1} \frac{q^{p(p-1) / 2-j p+j}(-q ; q)_{j}}{[j]_{q}} \\
& \equiv-\frac{1}{[p]_{q}} \sum_{j=1}^{p-1}(-1)^{j} q^{\left(\begin{array}{c}
p \\
2
\end{array}\right)+\left(\begin{array}{c}
j \\
2
\end{array}\right)-j p+j}\left[\begin{array}{c}
p \\
j
\end{array}\right]_{q}(-q ; q)_{j} \\
& =-\frac{1}{[p]_{q}} \sum_{j=1}^{p-1}(-1)^{j} q^{\left(\begin{array}{c}
p-j \\
2
\end{array}\right)}\left[\begin{array}{c}
p \\
j
\end{array}\right]_{q}(-q ; q)_{j} \\
& =-\frac{1}{[p]_{q}}\left((-1)^{p} q^{\left(\begin{array}{c}
p+1 \\
2
\end{array}\right)}-q^{\left(\begin{array}{c}
p \\
2
\end{array}\right)}-(-1)^{p}(-q ; q)_{p}\right)\left(\bmod [p]_{q}\right) .
\end{aligned}
$$

Notice that

$$
\frac{2-q^{\left(\begin{array}{c}
p+1 \\
2
\end{array}\right)}-q^{\left(\begin{array}{c}
p \\
2
\end{array}\right)}}{[p]_{q}} \equiv \frac{p+1}{2}(1-q)+\frac{p-1}{2}(1-q)=p(1-q)\left(\bmod [p]_{q}\right),
$$

and that

$$
\begin{aligned}
& \frac{(-q ; q)_{p}-2}{[p]_{q}} \\
& \quad=\frac{(-q ; q)_{p-1}\left(1+q^{p}\right)-2}{[p]_{q}}=\frac{2(-q ; q)_{p-1}-2}{[p]_{q}}-(1-q)(-q ; q)_{p-1} \\
& \quad \equiv 2 \mathrm{Q}_{p}(2, q)-(1-q)\left(\bmod [p]_{q}\right) .
\end{aligned}
$$

Hence

$$
\begin{aligned}
\sum_{j=1}^{p-1} \frac{q^{j}(-q ; q)_{j}}{[j]_{q}} & \equiv-\frac{(-q ; q)_{p}-q^{\left(\begin{array}{c}
p+1 \\
2
\end{array}\right)}-q^{\left(\begin{array}{c}
p \\
2
\end{array}\right)}}{[p]_{q}} \\
& \equiv-2 \mathrm{Q}_{p}(2, q)-(p-1)(1-q)\left(\bmod [p]_{q}\right)
\end{aligned}
$$

REMARK. Corollary 5.3 is the $q$-analogue of an observation of Glaisher:

$$
\frac{2^{p-1}-1}{p} \equiv-\sum_{j=1}^{p-1} \frac{2^{j-1}}{j}(\bmod p) \text {. }
$$

Lemma 5.4.

$$
\sum_{k=1}^{n}(-1)^{k}\left[\begin{array}{l}
n \\
k
\end{array}\right]_{q} q^{\left(\begin{array}{c}
n-k \\
2
\end{array}\right)} \frac{(-q ; q)_{k}}{[k]_{q}}=q^{\left(\begin{array}{c}
n \\
2
\end{array}\right)} \sum_{k=1}^{n} \frac{(-q)^{k}-1}{[k]_{q}} .
$$

Proof. We make an induction on $n$. The case $n=1$ is trivial. Assume that $n>1$ and that (5.6) holds for the smaller values of $n$. Then we conclude 
that

$$
\begin{aligned}
& \sum_{k=1}^{n}(-1)^{k}\left[\begin{array}{l}
n \\
k
\end{array}\right]_{q} q^{\left(\begin{array}{c}
n-k \\
2
\end{array}\right)} \frac{(-q ; q)_{k}}{[k]_{q}} \\
& =\sum_{k=1}^{n}(-1)^{k}\left(q^{k}\left[\begin{array}{c}
n-1 \\
k
\end{array}\right]_{q}+\left[\begin{array}{c}
n-1 \\
k-1
\end{array}\right]_{q}\right) q^{\left(\begin{array}{c}
n-k \\
2
\end{array}\right)} \frac{(-q ; q)_{k}}{[k]_{q}} \\
& =q^{n-1} \sum_{k=1}^{n-1}(-1)^{k}\left[\begin{array}{c}
n-1 \\
k
\end{array}\right]_{q} q^{\left(\begin{array}{c}
n-k-1 \\
2
\end{array}\right)} \frac{(-q ; q)_{k}}{[k]_{q}} \\
& +\frac{1}{[n]_{q}} \sum_{k=1}^{n}(-1)^{k}\left[\begin{array}{l}
n \\
k
\end{array}\right]_{q} q^{\left(\begin{array}{c}
n-k \\
2
\end{array}\right)}(-q ; q)_{k} \\
& =q^{\left(\begin{array}{c}
n \\
2
\end{array}\right)} \sum_{k=1}^{n-1} \frac{(-q)^{k}-1}{[k]_{q}}+\frac{1}{[n]_{q}}\left((-1)^{n} q^{\left(\begin{array}{c}
n+1 \\
2
\end{array}\right)}-q^{\left(\begin{array}{c}
n \\
2
\end{array}\right)}\right),
\end{aligned}
$$

where in the last step we apply the induction hypothesis and Lemma 5.2.

Proof of Theorem 5.1. Using Lemma 5.4, we have

$$
\begin{aligned}
& \sum_{j=1}^{p-1} \frac{q^{j}(-q ; q)_{j}}{[j]_{q}^{2}} \equiv \sum_{j=1}^{p-1} \frac{q^{p(p-1) / 2-j p+j}(-q ; q)_{j}}{[j]_{q}^{2}} \\
& \equiv-\frac{1}{[p]_{q}} \sum_{j=1}^{p-1}(-1)^{j} q^{\left(\begin{array}{c}
p-j \\
2
\end{array}\right)}\left[\begin{array}{c}
p \\
j
\end{array}\right]_{q} \frac{(-q ; q)_{j}}{[j]_{q}} \\
& =-\frac{q^{\left(\begin{array}{l}
p \\
2
\end{array}\right)}}{[p]_{q}} \sum_{j=1}^{p} \frac{(-q)^{j}-1}{[j]_{q}}+(-1)^{p} \frac{(-q ; q)_{p}}{[p]_{q}^{2}} \\
& =-\frac{q^{\left(\begin{array}{l}
p \\
2
\end{array}\right)}}{[p]_{q}} \sum_{j=1}^{p-1} \frac{(-q)^{j}-1}{[j]_{q}}-\frac{(-q ; q)_{p}-q^{\left(\begin{array}{c}
p+1 \\
2
\end{array}\right)}-q^{\left(\begin{array}{c}
p \\
2
\end{array}\right)}}{[p]_{q}^{2}}\left(\bmod [p]_{q}\right) .
\end{aligned}
$$

With help of (1.3) and Theorem 1.1, we get

$$
\begin{aligned}
\sum_{k=1}^{p-1} \frac{(-q)^{j}-1}{[j]_{q}}=-\sum_{k=1}^{p-1} \frac{(-1)^{j}\left(1-q^{j}\right)}{[j]_{q}}+\sum_{k=1}^{p-1} \frac{(-1)^{j}-1}{[j]_{q}} \\
=-\sum_{j=1}^{(p-1) / 2} \frac{2}{[2 j-1]_{q}}=\sum_{j=1}^{(p-1) / 2} \frac{2}{[2 j]_{q}}-\sum_{k=1}^{p-1} \frac{2}{[j]_{q}} \\
\equiv-2 \mathrm{Q}_{p}(2, q)+\mathrm{Q}_{p}(2, q)^{2}[p]_{q}+\mathrm{Q}_{p}(2, q)(1-q)[p]_{q}+\frac{p^{2}-1}{8}(1-q)^{2}[p]_{q} \\
\quad-\left((p-1)(1-q)+\frac{p^{2}-1}{12}(1-q)^{2}[p]_{q}\right)\left(\bmod [p]_{q}^{2}\right)
\end{aligned}
$$


By (2.4) we have

$$
\begin{aligned}
& \frac{(-q ; q)_{p}-q^{\left(\begin{array}{c}
p+1 \\
2
\end{array}\right)}-q^{\left(\begin{array}{c}
p \\
2
\end{array}\right)}}{[p]_{q}^{2}} \\
& =\frac{2(-q ; q)_{p-1}-q^{\left(\begin{array}{c}
p+1 \\
2
\end{array}\right)}-q^{\left(\begin{array}{c}
p \\
2
\end{array}\right)}}{[p]_{q}^{2}}-\frac{(-q ; q)_{p-1}}{[p]_{q}}(1-q) \\
& \equiv \frac{2(-q ; q)_{p-1}-2}{[p]_{q}^{2}}+\frac{p}{[p]_{q}}(1-q)-\frac{(p-1)^{2}}{4}(1-q)^{2}-\frac{(-q ; q)_{p-1}}{[p]_{q}}(1-q) \\
& =\frac{2 \mathrm{Q}_{p}(2, q)}{[p]_{q}}+\frac{(p-1)(1-q)}{[p]_{q}}-\frac{(p-1)^{2}}{4}(1-q)^{2} \\
& \quad-\mathrm{Q}_{p}(2, q)(1-q)\left(\bmod [p]_{q}\right) .
\end{aligned}
$$

Therefore

$$
\begin{aligned}
& \sum_{j=1}^{p-1} \frac{q^{j}(-q ; q)_{j}}{[j]_{q}^{2}} \\
& \equiv\left(\frac{(p-1)(1-q)}{2}-\frac{1}{[p]_{q}}\right) \sum_{j=1}^{p-1} \frac{(-q)^{j}-1}{[j]_{q}}-\frac{(-q ; q)_{p}-q^{\left(\begin{array}{c}
p+1 \\
2
\end{array}\right)}-q^{\left(\begin{array}{c}
p \\
2
\end{array}\right)}}{[p]_{q}^{2}} \\
& \equiv-(p-1) \mathrm{Q}_{p}(2, q)(1-q)-\mathrm{Q}_{p}(2, q)^{2} \\
& \quad-\frac{(7 p-5)(p-1)}{24}(1-q)^{2}\left(\bmod [p]_{q}\right) .
\end{aligned}
$$

Acknowledgements. I am grateful to the anonymous referee for his/her helpful suggestions. I also thank my advisor, Prof. Zhi-Wei Sun, for his useful comments.

\section{References}

[AKS] M. Agrawal, N. Kayal and N. Saxena, PRIMES is in P, Ann. of Math. 160 (2004), 781-793.

[A] G. E. Andrews, q-Analogs of the binomial coefficient congruences of Babbage, Wolstenholme and Glaisher, Discrete Math. 204 (1999), 15-25.

[AAR] G. E. Andrews, R. Askey and R. Roy, Special Functions, Cambridge Univ. Press, Cambridge, 1999.

[CP] R. Chapman and H. Pan, q-Analogues of Wilson's theorem, Int. J. Number Theory, accepted.

[C] W. E. Clark, q-Analogue of a binomial coefficient congruence, Int. J. Math. Math. Sci. 18 (1995), 197-200.

[F] R. D. Fray, Congruence properties of ordinary and q-binomial coefficients, Duke Math. J. 34 (1967), 467-480. 
[G1] A. Granville, Arithmetic properties of binomial coefficients I: Binomial coefficients modulo prime powers, in: Organic Mathematics (Burnaby, BC, 1995), CMS Conf. Proc. 20, Amer. Math. Soc., Providence, RI, 1997, 253-276.

[G2] - , The square of the Fermat quotient, Integers 4 (2004), A22.

[GZ] V. J. W. Guo and J. Zeng, Some arithmetic properties of the q-Euler numbers and q-Salié numbers, European J. Combin. 27 (2006), 884-895.

[Leh] E. Lehmer, On congruences involving Bernoulli numbers and the quotients of Fermat and Wilson, Ann. of Math. 39 (1938), 350-360.

[Ler] M. Lerch, Zur Theorie des Fermatschen Quotienten $\left(a^{p-1}-1\right) / p=q(a)$, Math. Ann. 60 (1905), 471-490.

[M] F. Morley, Note on the congruence $2^{4 n} \equiv(-1)^{n}(2 n) ! /(n !)^{2}$, where $2 n+1$ is a prime, Ann. of Math. 9 (1895), 168-170.

[PS] H. Pan and Z. W. Sun, On q-Euler numbers, q-Salié numbers and q-Carlitz numbers, Acta Arith. 124 (2006), 41-57.

[S] Z. W. Sun, Products of binomial coefficients modulo $p^{2}$, ibid. 97 (2001), 87-98.

[SP] L.-L. Shi and H. Pan, A q-analogue of Wolstenholme's harmonic series congruence, Amer. Math. Monthly 114 (2007), 529-531.

[W] J. Wolstenholme, On certain properties of prime numbers, Quart. J. Math. 5 (1862), 35-39.

Department of Mathematics

Shanghai Jiaotong University

Shanghai 200240, People's Republic of China

E-mail: haopan79@yahoo.com.cn

Received on 20.6.2006

and in revised form on 28.3.2007 